\title{
Machine Learning the Voltage of Electrode Materials in Metal-ion Batteries
}

\author{
Rajendra P. Joshi, ${ }^{\dagger} \ddagger$ Jesse Eickholt, ${ }^{\ddagger}$ Liling Li, ${ }^{\ddagger}$ Marco Fornari, ${ }^{\dagger}$ Veronica \\ Barone, $^{*, \dagger}$ and Juan E. Peralta ${ }^{\dagger}$ \\ $\dagger$ Department of Physics and Science of Advanced Materials Program, Central Michigan \\ University, Mount Pleasant, MI, 48859, USA \\ $\ddagger$ Department of Computer Science, Central Michigan University, Mount Pleasant, MI, \\ 48859, USA \\ E-mail: v.barone@cmich.edu
}

\begin{abstract}
Machine learning (ML) techniques have rapidly found applications in many domains of materials chemistry and physics where large data sets are available. Aiming to accelerate the discovery of materials for battery applications, in this work, we develop a tool (http://se.cmich.edu/batteries) based on ML models to predict voltages of electrode materials for metal-ion batteries. To this end, we use deep neural network, support vector machine, and kernel ridge regression as ML algorithms in combination with data taken from the Materials Project Database, as well as feature vectors from properties of chemical compounds and elemental properties of their constituents. We show that our ML models have predictive capabilities for different reference test sets and, as an example, we utilize them to generate a voltage profile diagram and compare it to density functional theory calculations. In addition, using our models, we propose nearly 5,000 candidate electrode materials for Na- and K-ion batteries. We also make
\end{abstract}


available a web-accessible tool that, within a minute, can be used to estimate the voltage of any bulk electrode material for a number of metal-ions. These results show that $\mathrm{ML}$ is a promising alternative for computationally demanding calculations as a first screening tool of novel materials for battery applications.

\section{Keywords}

Machine learning, batteries, intercalation electrodes, web-tool, voltage predictor, voltage profile diagram

\section{Introduction}

Lithium-ion batteries (LIBs) have revolutionized the energy storage technology ${ }^{12}$ and played a crucial role in transforming portable devices in terms of performance, lifetime, weight, and size. They have also opened unprecedented possibilities for new greener technologies, for instance in the automotive sector. ${ }^{213}$ Despite currently being the dominating energy source for small energy scale devices, the transferability of traditional LIBs to higher energy scales remains a challenge mainly because of their relatively low energy density. ${ }^{4 / 7}$ Moreover, the future of large scale applications of LIBs is uncertain due to the scarcity of Li as a raw material, its increasing price, skyrocketing energy demand, and safety concerns. $\frac{8}{10}$ These issues call for more sustainable, cheaper, and better performing alternatives to the present technology.

Several new designs for metal-ion batteries have been proposed in the literature, including monovalent Na- and K-ion batteries, and multivalent Mg-, Ca-, and Al-ion batteries. $\stackrel{3}{5}$ |11 13 The development of these batteries has been limited mainly because of a lack of suitable electrode and electrolyte materials, stimulating considerable work devoted to enhancing their robustness. $\frac{14}{14}$ For electrode materials, the number of possible compounds that could intercalate metal ions such as $\mathrm{Li}, \mathrm{Na}, \mathrm{K}, \mathrm{Mg}, \mathrm{Ca}$, and $\mathrm{Al}$ is likely in the order of thousands; 
however, the majority of these materials remain unexplored as electrode components because of the experimental and computational difficulties to screen the large chemical and structural space with appropriate accuracy. $\frac{15}{17}$ In this context, data driven machine learning (ML) approaches provide ways to address this issue faster and with limited use of computational resources. $18 \mid 19$

ML-based search for new electrode materials requires a sufficient amount of well curated and verified data. ${ }^{20}$ Recently, enabled by the significant improvement in computing architectures, several databases based on density functional theory (DFT) calculations such as AFLOW, $\stackrel{2122}{2}$ Materials Project, $\stackrel{23 \mid 24}{ }$ OQMD, $\stackrel{25126}{2}$ and NOMAD ${ }^{27}$ were made readily available to the scientific community. Although DFT predictions are not the gold standard for theoretical calculations in some contexts, $\frac{28}{28}$ they do provide reasonable insights and can be used to guide experimental research. ${ }^{29}$ In several cases, these electronic structure databases have been combined with ML approaches for predicting specific properties of interest in tar-

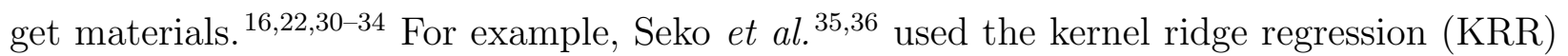
and support vector regression (SVR) to predict the cohesive energy of binary and ternary compounds, thermal conductivity of binary inorganic compounds, and melting temperature of single- and binary-component solids. Similarly, Meredig et al. ${ }^{37}$ built a ML model to predict the thermodynamic stability of any arbitrary chemical composition and proposed nearly 4500 new stable chemical compounds. ML approaches have also been used to improve the quality of exchange-correlation functionals in DFT. 180 These approaches have also been applied for the prediction of the band gap, total energy, and to find the potential candidate materials for photovoltaic cells, glass alloys, etc. $\stackrel{41}{43}$ Logistic regression was used by Sendek et $a l . \underline{44}$ to screen nearly 12,000 solids containing Li to propose new materials for electrolytes in Li-ion batteries. Similarly, online tools for ML predictions of electronic, thermal, and mechanical properties have been made available lately. 22

In this work, we employ ML to develop a tool to predict electrode voltages for metal-ion batteries using data from the Materials Project database. One of the challenges associated 
with a ML approach in materials science is the problem of finding the proper feature vectors that can accurately represent the compounds. $\frac{45}{47}$ In the literature, feature vectors derived from several approaches have been used. $\stackrel{46}{49}$ A simple, yet effective, approach is the one derived from chemical, structural, elemental, and electronic representation of the compounds. $\underline{46}$ Here, we utilize the feature vectors derived from the chemical properties of compounds and the properties of their elemental constituents in combination with deep neural networks $(\mathrm{DNN}), \stackrel{50151}{ }$ support vector machine $(\mathrm{SVM}), \underline{52}$ and kernel ridge regression (KRR). ${ }^{53}$ Voltage profile diagrams generated from ML methods are compared to the corresponding DFT based diagrams. We also provide a web-accessible interface that predicts the voltage of any electrode material (for any metal-ion battery) with minimal basic information and within minutes. Our work shows that ML models can be employed as an exploratory tool to predict the voltage of electrode materials very efficiently.

\section{Data and Feature Vectors}

Our training data were extracted from the Materials Project Database containing a total of 4,250 data instances for 3,580 intercalation based electrode materials. ${ }^{23124}$ We utilized the Materials Projects application programming interface pymatgen to access the data from the database. ${ }^{23124}$ Each data instance corresponds to the average voltage calculated for a material in-between two concentrations of intercalating metal-ion. For some electrodes, average voltages are calculated for multiple concentrations, resulting in more data instances in comparison to the number of electrode materials. A total of 3,977 data instances were kept after removing inconsistencies and repetitions. The dataset that we use contains DFT predicted voltages for several metal ion batteries such as $\mathrm{Li}, \mathrm{Mg}, \mathrm{Ca}, \mathrm{Al}, \mathrm{Zn}$, and Y. Nearly $65 \%$ of the data corresponds to Li-ion battery materials, while data for $\mathrm{Ca}, \mathrm{Mg}$, and Zn-ion batteries comprises $10 \%$ each in the database. Additionally, $4 \%$ of data instances correspond to Y and Al-ion batteries. The database provides the average voltage of materials starting from 
completely de-intercalated to fully intercalated (e.g. $\mathrm{C}_{6} \rightarrow \mathrm{LiC}_{6}$, considering $\mathrm{C}_{6}$ as electrode material and $\mathrm{Li}$ as the metal) as well as from partially intercalated to fully intercalated (e.g. $\left.\mathrm{Li}_{0.5} \mathrm{C}_{6} \rightarrow \mathrm{LiC}_{6}\right)$.

The features used to specify a particular electrode material in our ML models include the working ion in the battery (i.e. Li or other metal), the concentration of the active metal ion in a given compound, crystal lattice types, and space group numbers. All other features were obtained from the elemental properties of the atomic constituents involved in a particular electrode. The elemental properties added to the feature vectors are adopted from the work of Ward et al. $\underline{41}$ and are listed in the Supplementary Information (SI). This results in 237 features that uniquely represent each compound in the data set. The components of a feature vector are diverse in terms of their magnitudes which can range from a few thousands to small fractions. Therefore, all the input features were normalized for a better and more efficient training of the model that avoids any biased preference of a particular feature with respect to others based solely on their magnitude. Normalization was performed by effectively scaling all the inputs to be between -1 and 1, and it was carried out on-the-fly while training our model and it is not done for target values.

To systematically remove the redundancy within the features vectors and also to reduce the dimensionality of the features space, we used principal component analysis (PCA) algorithms as implemented in the sklearn library. ${ }^{56}$ In Figure 1, we show the cumulative explained variance as a function of the number of principal components (PC). It shows that only 80 PC are required, reducing the dimension of feature space significantly. Thus, only these 80 PC were fed into our ML models.

Our data were split into two parts, the training set (T-set, 90\%) and the holdout test set (H-set, 10\%). The T-set is randomly shuffled and used for parameter optimization with a 10 -fold cross validation. In the 10-fold cross validation, data is randomly divided into 10 equal segments (known as folds), and data in 9 of these folds are used for training the models and the remaining fold for validating the model. This is repeated 10 times in a way that each 


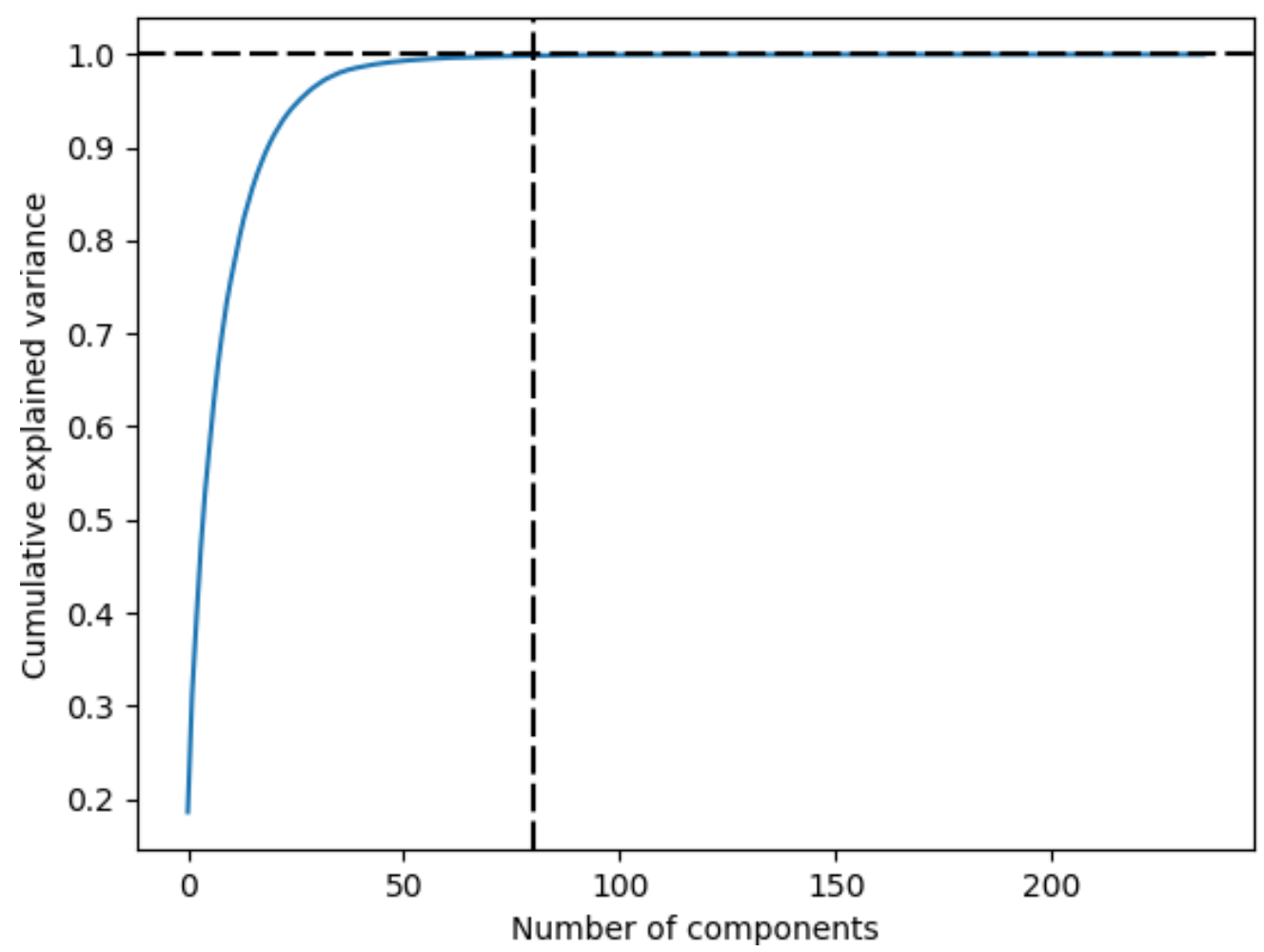

Figure 1: Principal component analysis for the feature vectors leads to a reduction of $66 \%$ of the dimensionality.

of the folds is used as validation set. Independent validation of the models was done with the $\mathrm{H}$-set which is not utilized during the training process. In addition, we also examined the performance of our models on a limited Na-ion batteries data set (Na-set) taken from the literature. ${ }^{57}$ To assess the performance of our machine learning models we utilize the mean absolute error (MAE) defined as

$$
\text { MAE }=\frac{1}{N} \sum_{i}^{N}\left|Y_{\text {target }}^{i}-Y_{\text {predicted }}^{i}\right|,
$$

where $Y_{\text {target }}^{i}$ and $Y_{\text {predicted }}^{i}$ are the target predicted values using our models for a given set of parameters for each material $i$, and $N$ is the number of instances in the data set. 


\section{Results and Discussion}

With the goal of assessing the performance of different ML algorithms, we have employed three machine learning algorithms: DNN, SVM and KRR. This assessment allows us to select the most robust model for our web-tool. A 4-layer DNN was used in our work with an architecture shown in Figure 2. To limit overfitting, we added a dropout layer $\frac{58}{\sqrt{58}}$ after the second and third layer with a rate of $25 \%$ and $10 \%$ respectively. In addition, we used L2 regularization technique in our work. $\frac{59}{}$ In the input layer, we have 80 nodes with 60 and 30 nodes in the first and second hidden layer, respectively, and one node in the output layer. All of these parameters were tuned for optimal performance of the model in the T-set and were chosen optimal when we obtained minimum MAE and when we did not detect signs of overfitting and underfitting. We have used mean square error as loss function and MAE as a metric to measure the performance of the model as implemented in the Keras machine learning library. $[\underline{60}$ We use "rmsprop" as an algorithm to optimize the loss function.

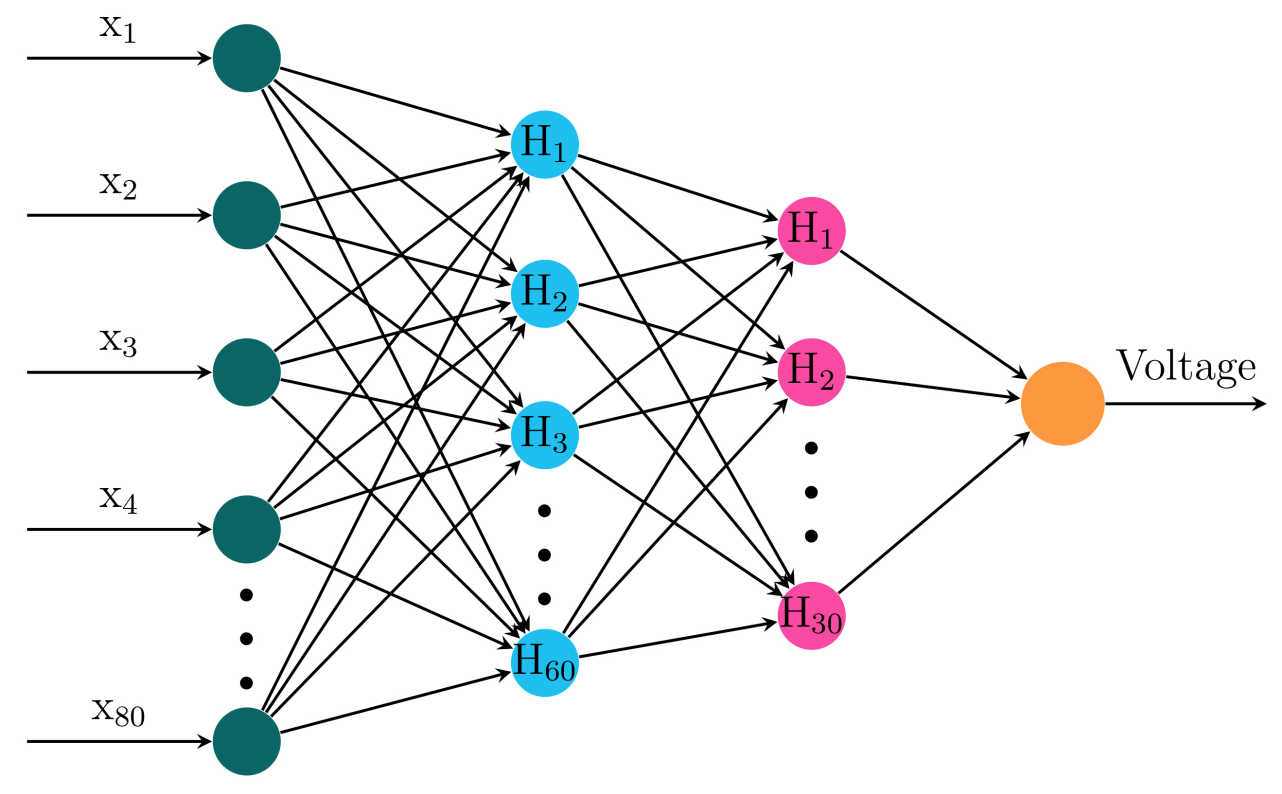

Figure 2: Architecture of the deep neural network used in our work. $\mathrm{x}_{i}$ are the inputs for the input layer and $\mathrm{H}_{i}$ represents nodes in hidden layers. The output from the output layer is the voltage.

The MAE and the standard deviation for each fold in the T-set (computed with the 
optimal parameters) are shown in Table 1. We obtained similar MAE in all 10 folds of the DNN model with mean MAE of $0.43 \mathrm{~V}$ and standard deviation of $\pm 0.03 \mathrm{~V}$. We obtained a similar MAE of $0.43 \mathrm{~V}$ in the $\mathrm{H}$-set. The performance of the model in the $\mathrm{H}$-set is shown in Figure 4(a), where a linear relationship between target and DNN predicted values is clearly shown.

Table 1: Mean absolute error (V) for each fold of 10-fold cross-validation using deep neural network (DNN), support vector regression (SVR) and kernel ridge regression (KRR). The mean and the standard deviation of the MAE in these 10 cross-validation sets is given. In addition, the MAE for the $\mathrm{H}$-set and Na-set are provided.

\begin{tabular}{llll}
\hline \hline Fold & DNN & SVR & KRR \\
\hline 1 & 0.42 & 0.51 & 0.54 \\
2 & 0.48 & 0.25 & 0.28 \\
3 & 0.42 & 0.26 & 0.27 \\
4 & 0.44 & 0.35 & 0.47 \\
5 & 0.44 & 0.38 & 0.43 \\
6 & 0.42 & 0.62 & 0.71 \\
7 & 0.43 & 0.43 & 0.42 \\
8 & 0.41 & 0.59 & 0.62 \\
9 & 0.45 & 0.53 & 0.57 \\
10 & 0.48 & 0.28 & 0.30 \\
\hline Mean MAE \pm standard deviation & $0.43 \pm 0.03$ & $0.42 \pm 0.13$ & $0.46 \pm 0.14$ \\
\hline MAE H-set & 0.43 & 0.40 & 0.39 \\
\hline MAE Na-set & 1.25 & 1.00 & 0.93 \\
\hline \hline
\end{tabular}

We compared the performance of DNN with another machine learning model, SVM. When used for a regression problem, SVM is known as a support vector regression (SVR). SVR is a kernel-based regression technique known for its robust performance in complex data representations. It works by mapping non-linearly separable data in real space to higher dimensional space via a kernel function. We have used the radial basis function (RBF) kernel for this work. In addition, SVR depends on two important parameters $(C$ and $\gamma$ ) that control the quality of the result. These parameters were tuned by using the grid search algorithm of sklearn. We varied $C$ and $\gamma$ logarithmically in between $10^{-5}$ and $10^{5}$ each. For each of the possible combinations of $C$ and $\gamma$, SVR computations were performed using a 10-fold cross-validation to calculate the mean of the MAE. The parameter space and 
MAE encoded as color, obtained from a grid search is shown in Figure 3(a). We determined optimal values of $C$ and $\gamma$ such that they yield the minimum MAE from the grid search, which corresponds to an optimal combination of $C=10.0$ and $\gamma=0.1$ for which we obtain a mean MAE of $0.42 \pm 0.13 \mathrm{~V}$ in the T-set. Further refining the grid in the small range around the optimal values of $C$ and $\gamma$ does not qualitatively influence our results. The MAE for each fold of 10-fold cross-validated T-set obtained with these tuned parameters and the RBF kernel are given in Table 1. The performance of SVR for the H-set is shown in Figure 4(b).
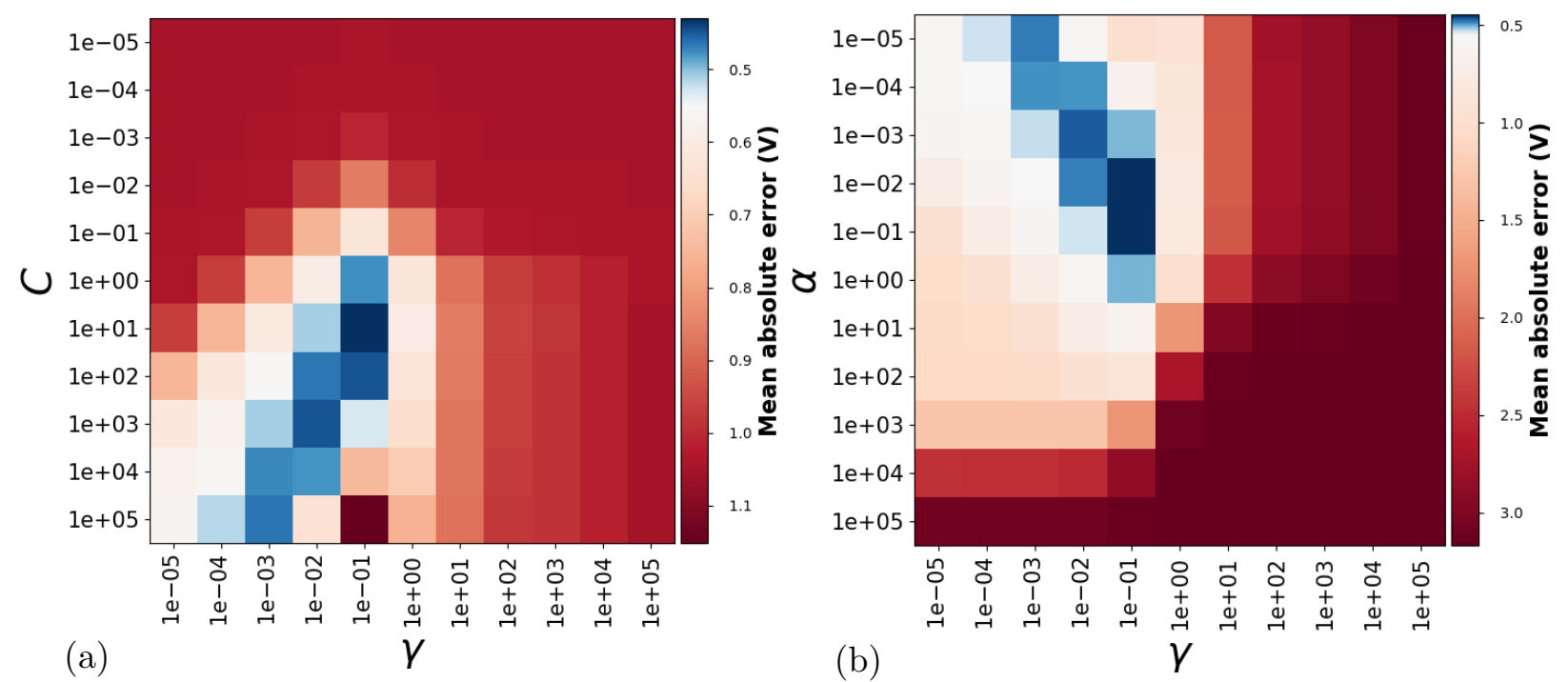

Figure 3: Color maps showing the tuning of (a) $C$ and $\gamma$ parameters for support vector regression and (b) $\alpha$ and $\gamma$ parameters for kernel ridge regression. The color bars display the mean absolute error.

In addition to SVR, the performance of DNN was compared with another kernel based ML algorithm known as kernel ridge regression (KRR). Similarly to SVR, we used a grid search technique to find the optimal parameters $\alpha$ and $\gamma$ by varying each between $10^{-5}$ and $10^{5}$, which is shown in Figure 3(b). In this case we obtained an optimal value of $\alpha$ and $\gamma$ as 0.01 and 0.1 , respectively, for which KRR yields a mean MAE of $0.46 \pm 0.14 \mathrm{~V}$ in T-set. The MAEs in the 10 folds of T-set for the KRR model are given in Table 1 alongside the values obtained from DNN and SVR. Finally, we checked the performance on the H-set, which is shown in Figure 4(c). We obtained a MAE of $0.39 \mathrm{~V}$ on the $\mathrm{H}$-set which is in agreement to what we observed using SVR $(0.40 \mathrm{~V})$. These MAEs for the H-set depend only marginally 

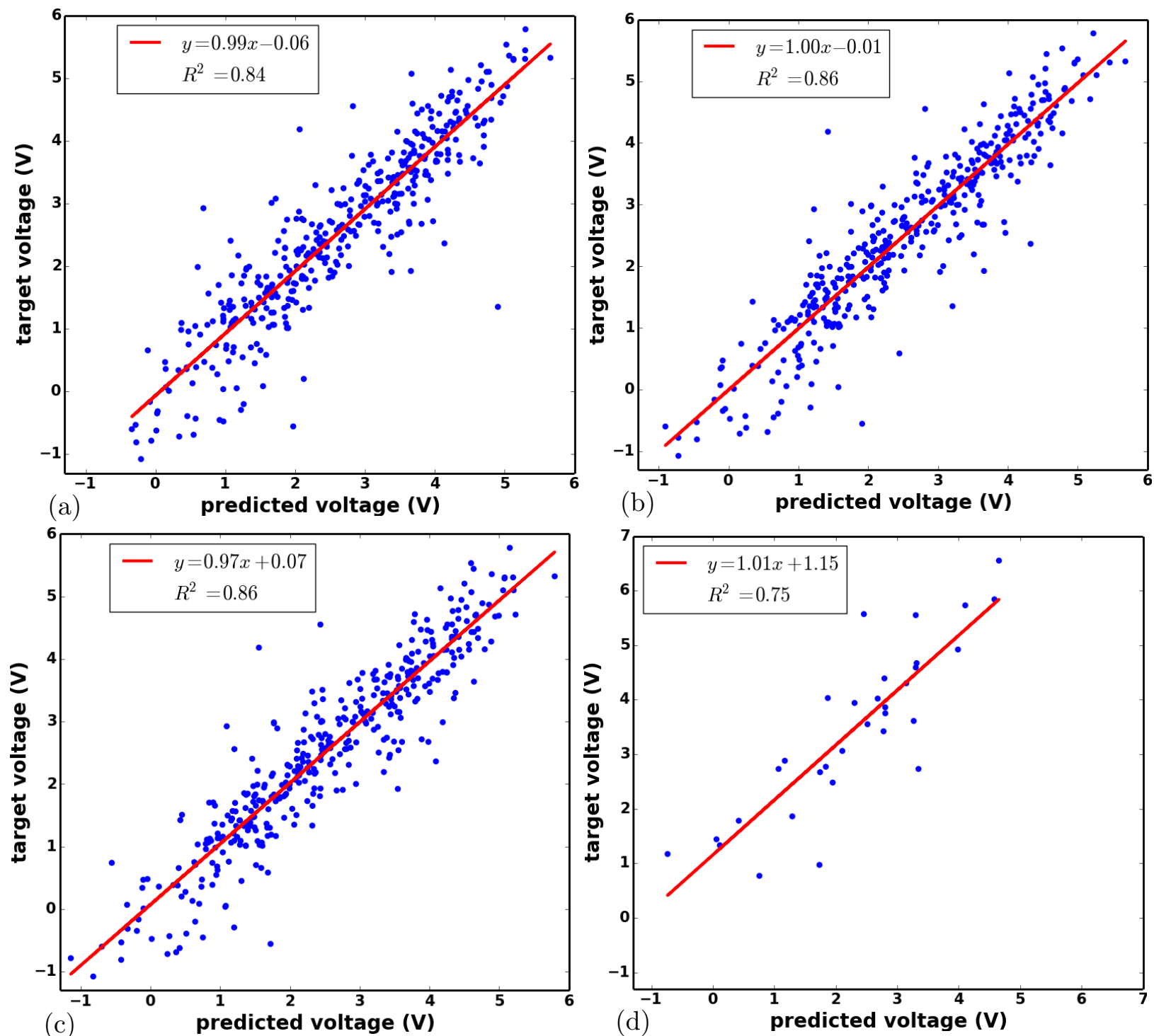

Figure 4: Scatter plot showing target vs. predicted voltages with different ML algorithms used in this work. (a) DNN, (b) SVR, (c) KRR, each on H-set. (d) DNN on Na-set. The best fit equation $(y=m x+c)$ and $R^{2}$ values for linear fit between target and ML predicted values are provided as an inset.

on the ML algorithms, although, with SVR and KRR, there are minor deviations between the folds related to the kernel based nature of both SVR and KRR: as we move between the folds, the data might not be that separable in folds when mapped using the kernel functions.

The performance of our ML models on the H-set is shown in Figure 4(a-c). We show in these scatter plots that for lower voltage ranges the models perform slightly worse than for the higher voltage range. We attribute this behavior to the limited amount of data available 
for training the model in this range. Such a limited amount of data might not be sufficient to capture the complexity of the patterns needed to predict voltages properly. We believe that the performance of the model in the low voltage region could be improved by adding more data points within this range to the T-set.

For each model, we also show in Figure $4(\mathrm{a}-\mathrm{c})$ the best-fit linear equation and the $R^{2}$ value as a measure of goodness of fit between the ML predicted and the target values in the hold out test set. In the ideal case, the equation of best fit should be $y=x$ whereas $R^{2}$ should be one. With our models, we observed this relationship between the target and the ML predicted values. In addition, the good performance of our models is also reflected in the $R^{2}$ values. We obtained reasonable $R^{2}$ values of $0.84,0.86$, and 0.86 with DNN, SVR, and KRR respectively.

We note that although our ML-models perform well and consistently, a more challenging test for the models would be achieved by gauging the performance of our models on a completely new data set. To this end, we checked the performance of the models on the Naset. This data set is taken from the work of Zhang et al. ${ }^{[57}$ and contains voltage information of 32 Na-battery electrode materials with voltages in the range of $0.7-6.5 \mathrm{~V}$. This set of materials was obtained by screening the entire Materials Project database for Na-based layered materials and then performing DFT calculations on the selected set of materials suitable as battery electrodes. The Na-set differs from the original T- and H-set as it contains the voltage for Na-based electrode materials (for which our model is not trained). Thus, this is an excellent test to examine the robustness and the transferability of our ML models. In Table 1, we list the MAE obtained for the Na-set with each of the ML models. In addition, in Figure 4(d) we show the scatter plots obtained with DNN. We observed that the model does not perform as well for Na-set as it does during cross validation for the T- and H-sets. We obtained a MAE about $0.8 \mathrm{~V}$ larger than in the case of the T- and H-set using DNN. Despite the large MAE, the linear relationship between the predicted and targets values is still preserved with our ML models. However, the poorer performance of the models for 
this test set is reflected in the relatively small $R^{2}$ value as well as the best fit line with large intercept. We identified two main reasons that could explain the comparatively poorer performance of our models in the Na-set. First, our model is not trained for Na-ion electrodes due to the lack of data in the databases for Na. Secondly, the Na-test set contains only 32 materials which can affect the statistical performance of the ML models.

\section{Li-only Data}

Our data set is diverse as it contains 6 different metal-ions ( $\mathrm{Li}, \mathrm{Ca}, \mathrm{Mg}, \mathrm{Zn}, \mathrm{Al}$, and $\mathrm{Y}$ ) battery electrodes in a relatively small amount of data. It is interesting to see how the ML models perform on a specific metal-ion battery data. As a representative case, and due to the availability of a relatively large proportion of data for Li-ion electrodes, we trained our ML models only on Li-only battery data (Li-set). The model parameters are tuned again with Li-only data using the same protocol as we used for the entire data (parameters are provided in the SI). The MAE obtained from all three ML models in 10-fold cross validated T-set, H-set, and Na-set are given in Table 2. We note that T-set and $\mathrm{H}$-set used in this

case are $90 \%$ and $10 \%$ of the Li-only data. The corresponding scatter plots in the H-set are provided in the SI.

Although the performance of the models in the T-set and $\mathrm{H}$-set, as quantified by the MAE, is similar to the one obtained considering the entire data set, the performance on the Na-set is improved in comparison to the models trained on the entire data set (see Table 1). Such an improvement might be due to the more coherent data for training the models as well as the similarity between Na-ion and Li-ion electrochemistry. In the case of models trained only on Li-data, a MAE of $0.62-0.70 \mathrm{~V}$ is obtained for the Na-set with all three models, which is slightly larger than what we obtained for the other test sets. This larger error is somewhat expected, and still promising, considering that the model is not trained for $\mathrm{Na}$ battery electrode materials and the small number statistics effect in the Na-set. Nonetheless, these results show that our ML models are transferable among different metal-ion batteries. 
Table 2: Mean absolute error (V) for each fold of 10-fold cross-validated T-set, H-set, and Na-set with DNN, SVR and KRR along with mean and standard deviation in MAE for the models trained on Li-set. Note that the T-set and H-set here are taken from the Li-only data set.

\begin{tabular}{llll}
\hline \hline Fold & DNN & SVR & KRR \\
\hline 1 & 0.44 & 0.65 & 0.64 \\
2 & 0.45 & 0.37 & 0.45 \\
3 & 0.53 & 0.30 & 0.34 \\
4 & 0.43 & 0.36 & 0.37 \\
5 & 0.48 & 0.43 & 0.42 \\
6 & 0.50 & 0.79 & 0.78 \\
7 & 0.48 & 0.47 & 0.46 \\
8 & 0.43 & 0.39 & 0.40 \\
9 & 0.48 & 0.68 & 0.73 \\
10 & 0.46 & 0.57 & 0.53 \\
\hline Mean MAE \pm standard deviation & $0.47 \pm 0.03$ & $0.50 \pm 0.15$ & $0.51 \pm 0.15$ \\
\hline MAE H-set & 0.42 & 0.44 & 0.44 \\
\hline MAE Na-set & 0.70 & 0.62 & 0.70 \\
\hline \hline
\end{tabular}

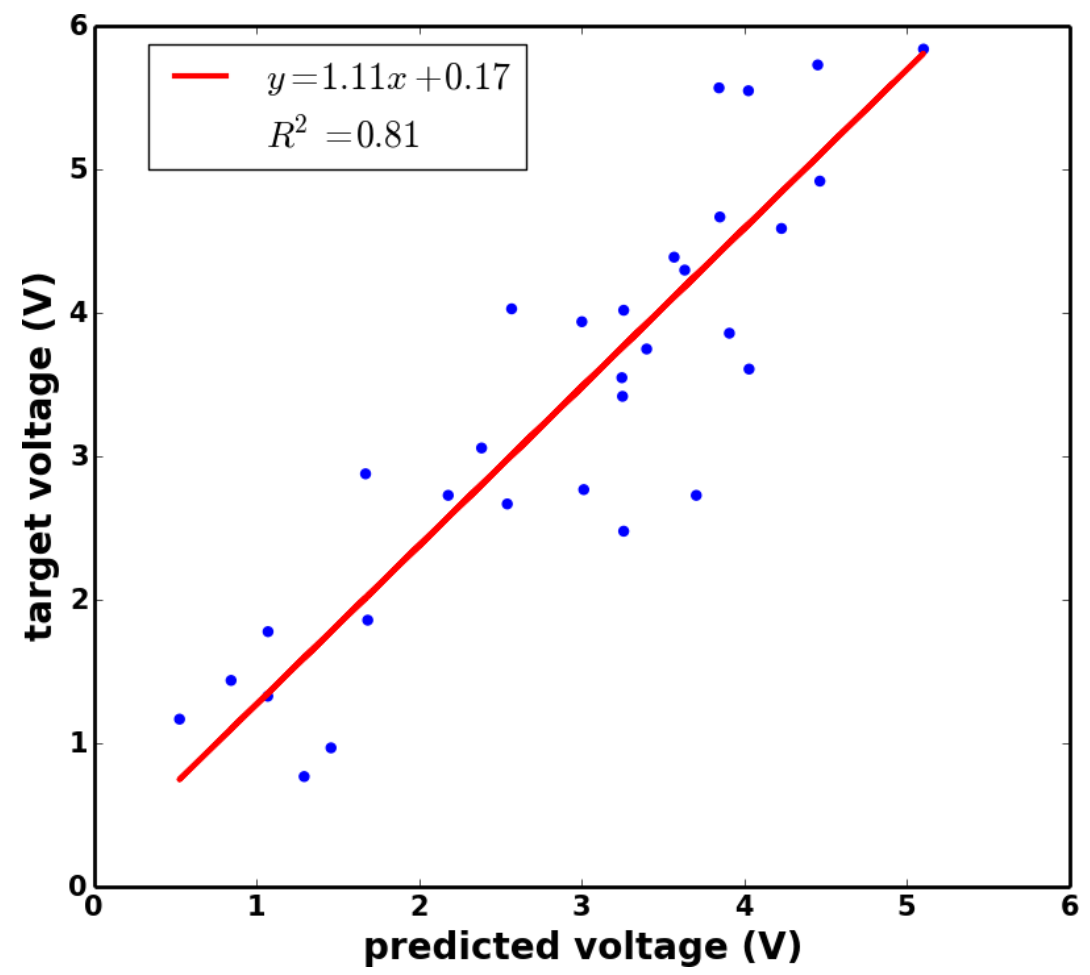

Figure 5: Scatter plot showing the target vs. the predicted values (V) with DNN trained on Li-set and applied to Na-set which is taken from the work of Zhang et al. $\frac{57}{\text { (other scatter }}$ plots are available in the SI). 
In Figure 5, we show the scatter plot obtained by using DNN on the Na-set. The performance of DNN on this new test set is similar to what we observed for the original Li-only training and test sets. The ML predicted voltage values follow a linear trend with respect to the target voltage. The improved performance on Na-set can also be seen from the best fit equation $(y=1.11 x+0.17$, compared to the entire data best fit equation $y=1.01 x+1.15$ on Na-set). Additionally, we observe an improvement in the $R^{2}$ value with the model trained only on Li data batteries for the Na-set. Similar results were obtained with SVR and KRR (the corresponding scatter plots are provided in the SI).

\section{Voltage Profiles}

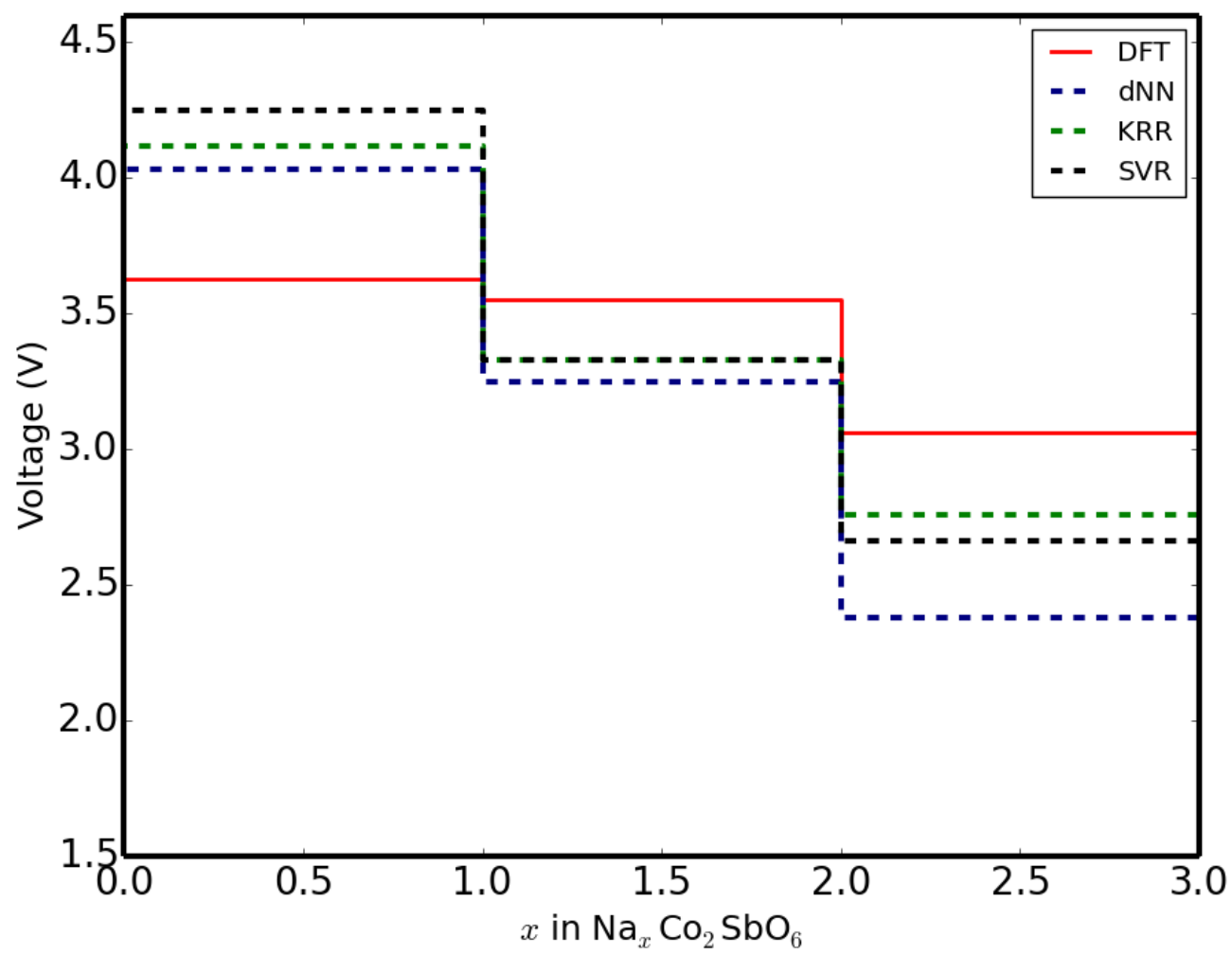

Figure 6: Voltage profile diagram obtained from different ML models and DFT for $\mathrm{Na}_{x} \mathrm{Co}_{2} \mathrm{SbO}_{6}$. The DFT values are taken from the work of Zhang et al.

The ML models we developed in this work can be used to generate the voltage profile diagram for electrode materials. These diagrams provide an estimate of the voltage range that an electrode can supply when used in a battery. To obtain these diagrams one needs 
to evaluate voltages at different intercalation concentrations. Our ML method offers a fast alternative to DFT methods estimate this voltages. As an example, in Figure 6 we show the voltage profile diagram of the Na-based electrode material $\mathrm{Na}_{x} \mathrm{Co}_{2} \mathrm{SbO}_{6}$, obtained from ML models and compare it with the one obtained using DFT, as reported by Zhang et al. ${ }^{[5]} \mathrm{In}$ the voltage profile diagrams, we observe that as the concentration of Na-ion increases, the DFT voltage decreases gradually. The same pattern is observed with the DNN predicted voltage in the electrode material considered. This trend is also reproduced by the other two machine learning algorithms (SVR and KRR) as shown in Figure 6 .

\section{Application: Prediction of New Electrodes}

The potential of our ML models to propose new electrode materials for Na- and K-ion batteries is assessed by studying candidate materials that are known for Li-ion but not necessarily explored for $\mathrm{Na}$ - and $\mathrm{K}$-ion batteries. In the literature, some of the materials used as electrode for Li-ion have also been studied for their use as electrodes in Na-ion batteries. For instance, Ceder et al. ${ }^{[1]}$ presented a comparative study for a set of selected cathode materials in terms of the voltage they offer when used as Li- and Na-ion battery electrodes. They found that a material provides on average $0.18-0.57 \mathrm{~V}$ lower voltage when used as electrode for Na-ion battery than the corresponding Li based electrodes, in agreement with experimental observations in some of these materials.

Although the generalization of their conclusion for all possible Li and $\mathrm{Na}$ based electrode materials is not documented, the trend observed from their study is encouraging as it implies that electrode materials that have been known over the last two decades for their use in Liion batteries can potentially work as electrodes in Na- and K-ion batteries operating at lower voltages. The majority of Li-based electrode materials existing in the MP-database are not

explored for Na- and K-ion battery electrodes. A quantitative estimate of their performance for Na- and K-ion batteries is necessary to build a robust and cheaper alternative to Liion batteries. Here, we use our ML tool to examine the performance of Li-based electrode 
materials found in the MP-database for $\mathrm{Na}$ and $\mathrm{K}$ by replacing the intercalating Li-ion with Na- and K-ions. We utilize the same stoichiometry and crystal structure for $\mathrm{Na}$ and $\mathrm{K}$ intercalated material as in the parent Li-based material. The goal of this exercise is to propose new cathodes for Na- and K-ion batteries, predict their voltage and study the correlation of predicted voltage with respect to that of Li-ion.

We first verify the conclusion of Ceder et al. ${ }^{61}$ by taking a few materials from their work and calculating the voltage of these materials for Li-, Na-, and K-ions using our DNN ML model. These results are shown in Table 3, where we include DFT and experimental values when available. Our ML model reproduced the trend observed from DFT calculations. ML predicted voltages for Na-ion materials are on average $0.30 \mathrm{~V}$ smaller than the corresponding Li values. This is comparable to the $0.40 \mathrm{~V}$ shift observed from DFT calculations for the same materials. The voltages predicted for K-ion materials are even smaller than the corresponding voltages in Na-ion materials. Overall, average voltages follow the trend $\mathrm{Li}>\mathrm{Na}>\mathrm{K}$.

Table 3: ML predicted voltage (V) for Li, Na and K-based electrodes. Corresponding DFT and experimental values (when available) are provided in parenthesis. DFT voltages, experimental references, and materials were taken from the work of Ceder et al. $\underline{61}$

\begin{tabular}{llll}
\hline \hline Material & $\mathrm{A}=\mathrm{Li}$ & $\mathrm{A}=\mathrm{Na}$ & $\mathrm{A}=\mathrm{K}$ \\
\hline $\mathrm{ACoO}_{2}$ & $3.56(3.99,4.10)$ & $3.40(3.48,2.80)$ & 3.26 \\
$\mathrm{ANiO}_{2}$ & $3.81(3.82,3.85)$ & $3.61(3.31,3.00)$ & 3.12 \\
$\mathrm{ATiO}_{2}$ & $1.96(1.94)$ & $1.79(1.37,>1.50)$ & 1.51 \\
$\mathrm{ATiS}_{2}$ & $1.71(1.82)$ & $1.66(1.64)$ & 1.53 \\
$\mathrm{AFePO}_{4}$ & $3.36(3.45,3.50)$ & $2.95(3.08,3.00)$ & 2.39 \\
$\mathrm{AMnPO}_{4}$ & $3.66(3.89,4.10)$ & $3.30(3.59)$ & 2.69 \\
$\mathrm{ACoPO}_{4}$ & $4.03(4.64,4.8)$ & $3.53(4.19)$ & 2.85 \\
$\mathrm{ANiPO}_{4}$ & $4.50(5.06,5.3)$ & $4.12(4.58)$ & 3.28 \\
$\mathrm{~A}_{3} \mathrm{~V}_{2}\left(\mathrm{PO}_{4}\right)_{3}$ & $3.54(3.13,3.8)$ & $3.11(2.94)$ & 2.59 \\
\hline
\end{tabular}

The voltage of new electrode materials based on Na- and K-ions is shown in Figure 7(a), where we show the scatter plot of the ML predicted voltages of Na-based electrodes against the DFT predicted voltage of Li-based electrodes for the same materials. For the majority of the materials studied, the conclusion of Ceder et al. remains valid: ML predicted voltages for Na-based electrodes are, in general, smaller than the corresponding Li ones. We obtained 
a mean difference and mean absolute difference between DFT Li-voltages and ML-predicted Na-voltages of $0.36 \mathrm{~V}$ and $0.56 \mathrm{~V}$, respectively.

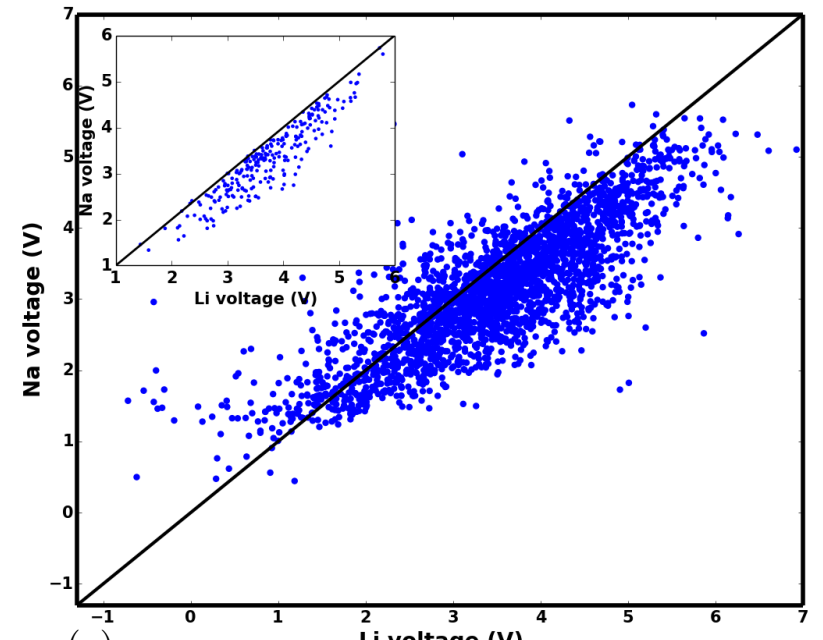

(a)

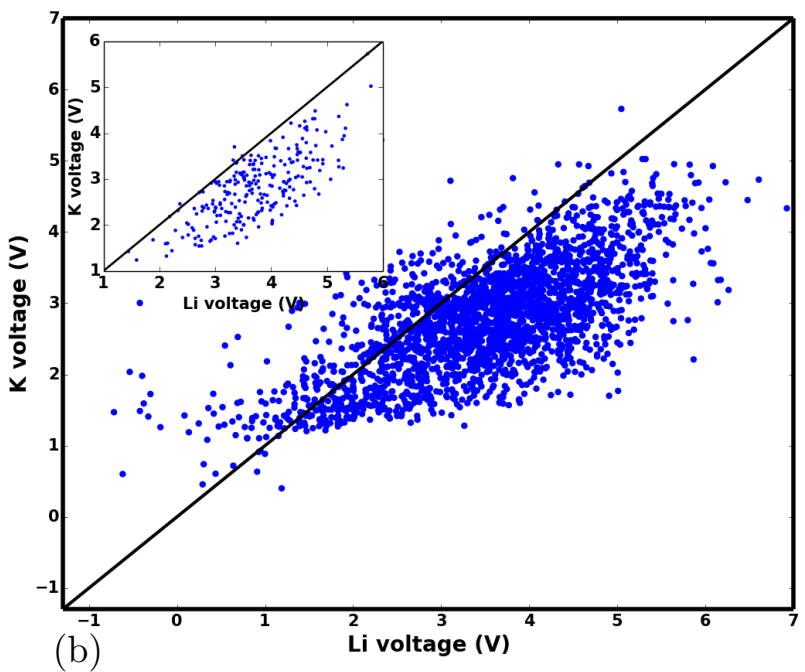

Figure 7: Scatter plots showing ML predicted voltages for electrode materials obtained by replacing Li ions with (a) $\mathrm{Na}$ ions and (b) $\mathrm{K}$ ions with respect to the corresponding DFT Li-voltages. The inset shows the ML predicted voltages for $\mathrm{Na}$ and $\mathrm{K}$-ion in the $\mathrm{H}$-set of Li-only materials with the corresponding ML-predicted voltages for Li-ion. The $y=x$ line is drawn as a reference for the eye.

For several electrodes, however, a larger voltage is predicted by our model for Na-based electrodes compared to the corresponding Li-based DFT values. This overestimation might arise because we are comparing ML-predicted voltages of Na-ion against DFT voltages of Li-ion. A better comparison for such correlation, perhaps, should consider ML-predicted voltages for both ions. To this end, we compared the ML-predicted voltage for the H-set used in the Li-only case for both Li- and Na-ion. The resulting scatter plot is shown in the inset of Figure 7(a). In this case, all the predicted voltages for Na-electrodes are smaller than the corresponding ones for Li-electrodes. The corresponding scatter plots for DFT Li-voltage and ML-predicted voltage for $\mathrm{Na}$ and K-based electrode materials in the H-set is provided in the SI (Figure S4). We obtained a mean deviation of $0.42 \mathrm{~V}$ in the H-set, which is consistent with the trend observed by Ceder et al. 61

A similar analysis was performed for K-based electrodes. We observed that voltages calculated for K-ion are even smaller than in Na- and Li-ions. The corresponding scatter 
plot is given in Figure 7(b). ML predicted K-voltages are in general smaller by $0.73 \mathrm{~V}$ compared to DFT Li-voltages. With respect to Li-electrodes in the H-set, ML predicted $\mathrm{K}$-voltages are smaller on average by $0.91 \mathrm{~V}$. The ML-predicted voltages for Na- and Kbased electrodes are provided in the SI along with the DFT voltages for the corresponding Li-electrodes.

With this analysis, we have identified a vast number of materials as well as their corresponding voltages that could work as suitable electrodes for Na- and K-ion batteries. From this first screening, researchers could further refine a set of materials in terms of structures, maximum metal stoichiometries, and voltages using DFT methods and, ultimately, experiments.

\section{Web Tool for Voltage Prediction}

We implemented a publicly available web-accessible tool for the battery community that can be used to predict the voltage of any novel electrode with minimal basic information typically within a minute. The only information required to predict the voltage are the stoichiometry of the intercalated material at high ion concentration, the stoichiometry of the material at low ion concentration, the type of metal-ion battery, the fraction of metal ions corresponding to the stoichiometry of the material at high ion concentration, the crystal lattice type, and the space group. This information is readily available for any material with a crystalline structure, making our tool efficient as well as remarkably easy to use. We used our most robust model, DNN, as a back-end ML model in our online tool for voltage prediction. Our tool can be used to predict the average voltage of Li-, Na-, K-, Mg-, Ca-, Zn-, Al- and Y-ion battery electrodes. Li-only trained model is used for prediction of voltage for battery electrodes based on Li-, Na-, and $\mathrm{K}$-ion whereas the entire data set trained model is used for rest of ions. This tool can be freely accessed at http://se.cmich.edu/batteries.

Knowing the performance of the web-tool in comparison to experimental reference might be of interest to the community exploring the electrodes material experimentally. As a proof 
Table 4: Table showing the DNN predicted vs experimental average voltage (V) for different electrodes.

\begin{tabular}{lll|lll}
\hline \hline Electrode & ML & Experiment & Electrode & ML & Experiment \\
\hline $\mathrm{NaMnO}_{2}$ & 2.98 & $2.75^{a}$ & $\mathrm{LiFePO}_{4}$ & 3.36 & $3.50^{b}$ \\
$\mathrm{NaCoO}_{2}$ & 3.40 & $2.80^{b}$ & $\mathrm{LiNiO}_{2}$ & 3.81 & $3.85^{b}$ \\
$\mathrm{NaTiO}_{2}$ & 1.79 & $>1.50^{b}$ & $\mathrm{NaFe}_{0.5} \mathrm{Co}_{0.5} \mathrm{O}_{2}$ & 3.58 & $3.14^{d}$ \\
$\mathrm{NaNiO}_{2}$ & 3.61 & $3.00^{b}$ & $\mathrm{~K}_{1.6} \mathrm{Na}_{2} \mathrm{Mnn}_{3} \mathrm{O}_{7}$ & 2.74 & $2.20^{e}$ \\
$\mathrm{LiCoO}_{2}$ & 3.60 & $4.10^{b}$ & $\mathrm{Mg}_{2} \mathrm{Mo}_{6} \mathrm{~S}_{8}$ & 1.09 & $1.30^{f}$ \\
$\mathrm{NaFePO}_{4}$ & 2.95 & $3.00^{b}$ & $\mathrm{Mg}_{0.55} \mathrm{TiSe}_{2}$ & 1.63 & $1.45^{g}$ \\
$\mathrm{Na}_{4} \mathrm{MnV}(\mathrm{PO})_{3}$ & 3.42 & $3.00^{c}$ & $\mathrm{MgMoO}_{3}$ & 2.20 & $2.25^{h}$ \\
\hline \hline
\end{tabular}

\begin{tabular}{l}
\hline \hline${ }^{a}$ taken from Ref. 62, \\
${ }^{b}$ taken from Ref. 61, \\
${ }^{c}$ taken from Ref. 63, \\
${ }^{d}$ taken from Ref. 64, \\
${ }^{e}$ taken from Ref. 65, \\
${ }^{f}$ taken from Ref. 666, \\
${ }^{g}$ taken from Ref. 67, \\
${ }^{h}$ taken from Ref. 68.,
\end{tabular}

of concept, we compare predicted average voltages with experimental average voltages. We selected few electrode material from the literature $\sqrt{61+63 \mid 65}$ and calculated their average voltage using the web-tool. These results are summarized in Table 4 where we observe a good agreement between the predicted voltages and the corresponding experimental values.

\section{Conclusions}

In conclusion, we used deep neural network, support vector regression, and kernel ridge regression to machine learn the voltage of electrode materials in a given metal-ion battery utilizing DFT data extracted from the Materials Project Database. The performance of the models is gauged by comparing the mean absolute error between the training set, the holdout test set, and data from the literature. Our results indicate that ML models reproduce DFT trends and thus can be used to explore electrode materials in terms of voltages. This methodology is fast compared to DFT calculations and can be used to guide experiments seeking to develop novel materials for battery applications or to perform a quick screening 
before starting synthesis procedures. Using our models, we propose nearly 5,000 electrode materials for Na- and K-ion batteries. Further improvement in the performance of the model might be necessary for routine application of ML algorithms for the prediction of voltage of electrode materials. Such improvements might include, but are not limited to, using other machine learning algorithms, using more data, or exploring other ways of feeding intercalation reactions to the ML models. In addition, for the robust training of ML models in the future, DFT calculations records of electrode materials in all voltage ranges should be kept in the databases (even if a material is not suitable as a battery electrode) since negative data-instances are also required for a proper training of data-intensive models. We also provide a web-accessible tool that can be used to predict the voltage of any electrode material within a minute.

\section{Acknowledgements}

We acknowledge the Material Project database for the data used in this work. R. P. J thanks Dr. Kai Trepte for careful proof reading of the manuscript. R. P. J also thanks Dr. Zhen Zhou and Dr. Xu Zhang for providing the DFT intercalation voltage for the voltage profile diagram. This work was supported by the Office of Basic Energy Sciences, US Department of Energy, de-sc0005027 and de-sc0019432.

\section{Supporting Information Available}

Scatter plots showing the performance of several ML models on the holdout test sets and the Na-test set, color map showing the parameter tuning in Li-only data, a table with the voltages predicted by DNN, SVM and KRR for Na-set along with the DFT values. A table with the ML predicted voltage of Na- and K-based electrodes along with the DFT values for Li-based electrodes. 


\section{References}

(1) Winter, M.; Barnett, B.; Xu, K. Before Li Ion Batteries. Chem. Rev. 2018, 118, 1143311456.

(2) Nitta, N.; Wu, F.; Lee, J. T.; Yushin, G. Li-ion Battery Materials: Present and Future. Materials Today 2015, 18, 252-264.

(3) Eames, C.; Islam, M. S. Ion Intercalation into Two-Dimensional Transition-Metal Carbides: Global Screening for New High-Capacity Battery Materials. J. Am. Chem. Soc. 2014, 136, 16270-16276.

(4) Joshi, R. P.; Ozdemir, B.; Barone, V.; Peralta, J. E. Hexagonal BC ${ }_{3}$ : A Robust Electrode Material for Li, Na, and K Ion Batteries. J. Phys. Chem. Lett. 2015, 6, 2728-2732.

(5) Bhauriyal, P.; Mahata, A.; Pathak, B. Hexagonal $\mathrm{BC}_{3}$ Electrode for a High-Voltage Al-Ion Battery. J. Phys. Chem. C 2017, 121, 9748-9756.

(6) Posada, J. O. G.; Rennie, A. J.; Villar, S. P.; Martins, V. L.; Marinaccio, J.; Barnes, A.; Glover, C. F.; Worsley, D. A.; Hall, P. J. Aqueous Batteries as Grid Scale Energy Storage Solutions. Renew. Sust. Energ. Rev. 2017, 68, 1174-1182.

(7) Dunn, B.; Kamath, H.; Tarascon, J.-M. Electrical Energy Storage for the Grid: a Battery of Choices. Science 2011, 334, 928-935.

(8) Liu, K.; Liu, Y.; Lin, D.; Pei, A.; Cui, Y. Materials for Lithium-Ion Battery Safety. Sci. $A d v .2018,4$.

(9) Tarascon, J.-M.; Armand, M. Materials For Sustainable Energy: A Collection of PeerReviewed Research and Review Articles from Nature Publishing Group; World Scientific, 2011; pp 171-179.

(10) Tarascon, J.-M. Is Lithium the New Gold? Nat. Chem. 2010, 2, 510. 
(11) Guduru, R. K.; Icaza, J. C. A Brief Review on Multivalent Intercalation Batteries with Aqueous Electrolytes. Nanomaterials 2016, 6, 41.

(12) Kulish, V. V.; Koch, D.; Manzhos, S. Ab initio Study of Li, Mg and Al Insertion into Rutile $\mathrm{VO}_{2}$ : Fast Diffusion and Enhanced Voltages for Multivalent Batteries. Phys. Chem. Chem. Phys. 2017, 19, 22538-22545.

(13) Thackeray, M. M.; Wolverton, C.; Isaacs, E. D. Electrical Energy Storage for Transportation-Approaching the Limits of, and going Beyond, Lithium-Ion Batteries. Energy Environ. Sci. 2012, 5, 7854-7863.

(14) Rajput, N. N.; Seguin, T. J.; Wood, B. M.; Qu, X.; Persson, K. A. In Modeling Electrochemical Energy Storage at the Atomic Scale; Korth, M., Ed.; Springer International Publishing: Cham, 2018; pp 79-124.

(15) Kirkpatrick, P.; Ellis, C. Chemical Space. Nature 2004, 432, 823.

(16) von Lilienfeld, O. A. Quantum Machine Learning in Chemical Compound Space. Angew. Chem. Int. Ed. 2018, 5\%, 4164-4169.

(17) Mullard, A. The Drug-maker's Guide to the Galaxy. Nature News 2017, 549, 445.

(18) Ghahramani, Z. Probabilistic Machine Learning and Artificial Intelligence. Nature 2015, 521, 452 .

(19) Jordan, M. I.; Mitchell, T. M. Machine Learning: Trends, Perspectives, and Prospects. Science 2015, 349, 255-260.

(20) Fornari, M.; Marzari, N. in The 2019 materials by design roadmap. J. Phys. D: Appl. Phys. 2018, 52, 013001.

(21) Curtarolo, S.; Setyawan, W.; Hart, G. L.; Jahnatek, M.; Chepulskii, R. V.; Taylor, R. H.; Wang, S.; Xue, J.; Yang, K.; Levy, O.; Mehl, M. J.; Stokes, H. T.; Dem- 
chenko, D. O.; Morgan, D. AFLOW: An Automatic Framework for High-Throughput Materials Discovery. Comput. Mater. Sci. 2012, 58, 218-226.

(22) Gossett, E.; Toher, C.; Oses, C.; Isayev, O.; Legrain, F.; Rose, F.; Zurek, E.; Carrete, J.; Mingo, N.; Tropsha, A.; Curtarolo, S. AFLOW-ML: A RESTful API for machinelearning predictions of materials properties. Comput. Mater. Sci. 2018, 152, 134-145.

(23) Jain, A.; Ong, S. P.; Hautier, G.; Chen, W.; Richards, W. D.; Dacek, S.; Cholia, S.; Gunter, D.; Skinner, D.; Ceder, G.; Persson, K. A. Commentary: The Materials Project: A Materials Genome Approach to Accelerating Materials Innovation. APL Mater. 2013, 1, 011002.

(24) Ong, S. P.; Cholia, S.; Jain, A.; Brafman, M.; Gunter, D.; Ceder, G.; Persson, K. A. The Materials Application Programming Interface (API): A Simple, Flexible and Efficient API for Materials Data based on REpresentational State Transfer (REST) principles. Comput. Mater. Sci. 2015, 97, 209-215.

(25) Saal, J. E.; Kirklin, S.; Aykol, M.; Meredig, B.; Wolverton, C. Materials Design and Discovery with High-Throughput Density Functional Theory: The Open Quantum Materials Database (OQMD). JOM 2013, 65, 1501-1509.

(26) Kirklin, S.; Saal, J. E.; Meredig, B.; Thompson, A.; Doak, J. W.; Aykol, M.; Rhl, S.; Wolverton, C. The Open Quantum Materials Database (OQMD): Assessing the Accuracy of DFT Formation Energies. npj Comput. Mater. 2015, 1, 15010.

(27) Draxl, C.; Scheffler, M. NOMAD: The FAIR concept for big data-driven materials science. MRS Bulletin 2018, 43, 676-682.

(28) Joshi, R. P.; Trepte, K.; Withanage, K. P. K.; Sharkas, K.; Yamamoto, Y.; Basurto, L.; Zope, R. R.; Baruah, T.; Jackson, K. A.; Peralta, J. E. Fermi-Löwdin Orbital SelfInteraction Correction to Magnetic Exchange Couplings. J. Chem. Phys. 2018, 149, 164101. 
(29) Kaloni, T. P.; Joshi, R. P.; Adhikari, N. P.; Schwingenschlgl, U. Band Gap Tunning in BN-doped Graphene Systems with High Carrier Mobility. Appl. Phys. Lett. 2014, 104, 073116 .

(30) Ramprasad, R.; Batra, R.; Pilania, G.; Mannodi-Kanakkithodi, A.; Kim, C. Machine Learning in Materials Informatics: Recent Applications and Prospects. npj Comput. Mater. 2017, 3, 54 .

(31) Bassman, L.; Rajak, P.; Kalia, R. K.; Nakano, A.; Sha, F.; Sun, J.; Singh, D. J.; Aykol, M.; Huck, P.; Persson, K.; Vashishta, P. Active Learning for Accelerated Design of Layered Materials. npj Comput. Mater. 2018, 4, 74.

(32) Zhang, Y.; Ling, C. A Strategy to Apply Machine Learning to Small Datasets in Materials Science. npj Comput. Mater. 2018, 4, 25.

(33) Butler, K. T.; Davies, D. W.; Cartwright, H.; Isayev, O.; Walsh, A. Machine Learning for Molecular and Materials Science. Nature 2018, 559, 547.

(34) Schleder, G. R.; Padilha, A. C. M.; Acosta, C. M.; Costa, M.; Fazzio, A. From DFT to Machine Learning: Recent Approaches to Materials Science A Review. J. Phys. Materials 2019,

(35) Seko, A.; Hayashi, H.; Nakayama, K.; Takahashi, A.; Tanaka, I. Representation of Compounds for Machine-Learning Prediction of Physical Properties. Phys. Rev. B 2017, 95,144110 .

(36) Seko, A.; Maekawa, T.; Tsuda, K.; Tanaka, I. Machine Learning with Systematic Density-Functional Theory Calculations: Application to Melting Temperatures of Single- and Binary-Component Solids. Phys. Rev. B 2014, 89, 054303.

(37) Meredig, B.; Agrawal, A.; Kirklin, S.; Saal, J. E.; Doak, J. W.; Thompson, A.; Zhang, K.; Choudhary, A.; Wolverton, C. Combinatorial Screening for New Materi- 
als in Unconstrained Composition Space with Machine Learning. Phys. Rev. B 2014, 89, 094104.

(38) Brockherde, F.; Vogt, L.; Li, L.; Tuckerman, M. E.; Burke, K.; Müller, K.-R. Bypassing the Kohn-Sham equations with machine learning. Nat. Commun. 2017, 8, 872.

(39) Mills, K.; Spanner, M.; Tamblyn, I. Deep Learning and the Schrödinger Equation. Phys. Rev. A 2017, 96, 042113.

(40) Li, L.; Snyder, J. C.; Pelaschier, I. M.; Huang, J.; Niranjan, U.-N.; Duncan, P.; Rupp, M.; Mller, K.-R.; Burke, K. Understanding Machine-Learned Density Functionals. Int. J. Quantum Chem. 2016, 116, 819-833.

(41) Ward, L.; Agrawal, A.; Choudhary, A.; Wolverton, C. A General-Purpose Machine Learning Framework for Predicting Properties of Inorganic Materials. npj Comput. Mater. 2016, 2, 16028.

(42) Faber, F. A.; Lindmaa, A.; von Lilienfeld, O. A.; Armiento, R. Machine Learning Energies of 2 Million Elpasolite $\left(A B C_{2} D_{6}\right)$ Crystals. Phys. Rev. Lett. 2016, 117, 135502.

(43) Deml, A. M.; O’Hayre, R.; Wolverton, C.; Stevanović, V. Predicting Density Functional Theory Total Energies and Enthalpies of Formation of Metal-nonmetal Compounds by Linear Regression. Phys. Rev. B 2016, 93, 085142.

(44) Sendek, A. D.; Yang, Q.; Cubuk, E. D.; Duerloo, K.-A. N.; Cui, Y.; Reed, E. J. Holistic Computational Structure Screening of more than 12,000 Candidates for Solid Lithiumion Conductor Materials. Energy Environ. Sci. 2017, 10, 306-320.

(45) Isayev, O.; Oses, C.; Toher, C.; Gossett, E.; Curtarolo, S.; Tropsha, A. Universal Fragment Descriptors for Predicting Properties of Inorganic Crystals. Nature Comm. 2017, 8, 15679 . 
(46) Ghiringhelli, L. M.; Vybiral, J.; Levchenko, S. V.; Draxl, C.; Scheffler, M. Big Data of Materials Science: Critical Role of the Descriptor. Phys. Rev. Lett. 2015, 114, 105503.

(47) Rupp, M.; von Lilienfeld, O. A.; Burke, K. Guest Editorial: Special Topic on DataEnabled Theoretical Chemistry. J. Chem. Phys. 2018, 148, 241401.

(48) Hansen, K.; Biegler, F.; Ramakrishnan, R.; Pronobis, W.; von Lilienfeld, O. A.; Mller, K.-R.; Tkatchenko, A. Machine Learning Predictions of Molecular Properties: Accurate Many-Body Potentials and Nonlocality in Chemical Space. J. Phys. Chem. Lett. 2015, 6, 2326-2331.

(49) Ward, L.; Liu, R.; Krishna, A.; Hegde, V. I.; Agrawal, A.; Choudhary, A.; Wolverton, C. Including Crystal Structure Attributes in Machine Learning Models of Formation Energies via Voronoi Tessellations. Phys. Rev. B 2017, 96, 024104.

(50) LeCun, Y.; Bengio, Y.; Gramfort, A.; Michel, V.; Thirion, B.; Grisel, O.; Hinton, G. Deep Learning. Nature 2015, 521, 436444.

(51) Goodfellow, I.; Bengio, Y.; Courville, A.; Bengio, Y. Deep Learning; MIT press Cambridge, 2016; Vol. 1.

(52) Noble, W. S. What is a Support Vector Machine? Nat. Biotechnol. 2006, 24, 15651567.

(53) Vovk, V. In Kernel Ridge Regression; B. Schoelkopf, V. V., Z. Luo, Ed.; Springer, 2013; pp 105-116.

(54) Pearson, K. LIII. On Lines and Planes of Closest Fit to Systems of Points in Space. Lond. Edinb. Dubl. Phil. Mag. 1901, 2, 559-572.

(55) Jolliffe, I. International encyclopedia of statistical science; Springer, 2011; pp 10941096. 
(56) Pedregosa, F.; Varoquaux, G.; Gramfort, A.; Grisel, O.; Michel, V.; Thirion, B.; Blondel, M.; Prettenhofer, P.; Weiss, R.; Dubourg, V.; Vanderplas, J.; Passos, A.; Cournapeau, D.; Brucher, M.; Perrot, M.; Duchesnay, E. Scikit-learn: Machine Learning in Python. J. Mach. Learn. Res. 2011, 12, 2825-2830.

(57) Zhang, X.; Zhang, Z.; Yao, S.; Chen, A.; Zhao, X.; Zhou, Z. An Effective Method to Screen Sodium-based Layered Materials for Sodium Ion Batteries. npj Comput. Mater. 2018, 4, 13 .

(58) Srivastava, N.; Hinton, G.; Krizhevsky, A.; Sutskever, I.; Salakhutdinov, R. Dropout: A Simple Way to Prevent Neural Networks from Overfitting. J. Mach. Learn. Res. 2014, 15, 1929-1958.

(59) Ng, A. Y. Feature Selection, L1 vs. L2 Regularization, and Rotational Invariance. Proceedings of the Twenty-first International Conference on Machine Learning. New York, NY, USA, 2004; p 78.

(60) Chollet, F. Keras: Deep learning library for theano and tensorflow. https://keras. io 2015, 7, T1.

(61) Ong, S. P.; Chevrier, V. L.; Hautier, G.; Jain, A.; Moore, C.; Kim, S.; Ma, X.; Ceder, G. Voltage, stability and diffusion barrier differences between sodium-ion and lithium-ion intercalation materials. Energy Environ. Sci. 2011, 4, 3680-3688.

(62) Billaud, J.; Clment, R. J.; Armstrong, A. R.; Canales-Vzquez, J.; Rozier, P.; Grey, C. P.; Bruce, P. G. $\beta-\mathrm{NaMnO}_{2}$ : A High-Performance Cathode for Sodium-Ion Batteries. J. Am. Chem. Soc. 2014, 136, 17243-17248.

(63) Nisar, U.; Shakoor, R.; Essehli, R.; Amin, R.; Orayech, B.; Ahmad, Z.; Kumar, P. R.; Kahraman, R.; Al-Qaradawi, S.; Soliman, A. Sodium Intercalation/de-intercalation Mechanism in $\mathrm{Na}_{4} \mathrm{MnV}\left(\mathrm{PO}_{4}\right)_{3}$ Cathode materials. Electrochim. Acta 2018, 292, 98 106. 
(64) Yoshida, H.; Yabuuchi, N.; Komaba, S. $\mathrm{NaFe}_{0.5} \mathrm{Co}_{0.5} \mathrm{O}_{2}$ as High Energy and Power Positive Electrode for Na-ion Batteries. Electrochem. Comm. 2013, 34, 60-63.

(65) Sada, K.; Senthilkumar, B.; Barpanda, P. Potassium-Ion Intercalation Mechanism in Layered $\mathrm{Na}_{2} \mathrm{Mn}_{3} \mathrm{O}_{7}$. ACS Appl. Energy Mater. 2018, 1, 5410-5416.

(66) Canepa, P.; Sai Gautam, G.; Hannah, D. C.; Malik, R.; Liu, M.; Gallagher, K. G.; Persson, K. A.; Ceder, G. Odyssey of Multivalent Cathode Materials: Open Questions and Future Challenges. Chem. Rev. 2017, 117, 4287-4341.

(67) Gu, Y.; Katsura, Y.; Yoshino, T.; Takagi, H.; Taniguchi, K. Rechargeable Magnesiumion Battery based on a TiSe ${ }_{2}$-Cathode with d-p orbital Hybridized Electronic Structure. Sci. Rep. 2015, 5, 12486.

(68) Gershinsky, G.; Yoo, H. D.; Gofer, Y.; Aurbach, D. Electrochemical and Spectroscopic Analysis of $\mathrm{Mg}^{2+}$ Intercalation into Thin Film Electrodes of Layered Oxides: $\mathrm{V}_{2} \mathrm{O}_{5}$ and $\mathrm{MoO}_{3}$. Langmuir 2013, 29, 10964-10972.

(69) Recham, N.; Rousse, G.; Sougrati, M. T.; Chotard, J.-N.; Frayret, C.; Mariyappan, S.; Melot, B. C.; Jumas, J.-C.; Tarascon, J.-M. Preparation and Characterization of a Stable $\mathrm{FeSO}_{4} \mathrm{~F}$-Based Framework for Alkali Ion Insertion Electrodes. Chem. Mater. 2012, 24, 4363-4370.

(70) Wang, L.; Zou, J.; Chen, S.; Zhou, G.; Bai, J.; Gao, P.; Wang, Y.; Yu, X.; Li, J.; Hu, Y.S.; Li, H. TiS 2 as a High Performance Potassium Ion Battery Cathode in Ether-based Electrolyte. Energy Storage Mater. 2018, 12, 216-222. 